\title{
A Non-Local Formulation of the Peierls Dislocation Model
}

\author{
Ron Miller and Rob Phillips, \\ Division of Engineering \\ Brown University \\ Providence, RI 02912, USA
}

\author{
Glenn Beltz, \\ Department of Mechanical and Environmental Engineering \\ University of California, Santa Barbara \\ Santa Barbara, CA 93106-5070, USA
}

and Michael Ortiz Graduate Aeronautical Labs California Institute of Technology Pasadena, CA 91125, USA

August 12, 2013 


\section{Abstract}

Cohesive zone models provide an illuminating and tractable way to include constitutive nonlinearity into continuum models of defects. Powerful insights have been gained by studying both dislocations and cracks using such analyses. Recent work has shown that as a result of the locality assumption present in such cohesive zone models, significant errors can be made in the treatment of defect energies. This paper aims to construct a non-local version of the Peierls-Nabarro model in which the atomic level stresses induced at the slip plane depend in a non-local way on the slip degrees of freedom. The non-local interplanar kernel is computed directly from atomistics and is used to evaluate both the structure and energetics of planar dislocations. The non-local formulation does not significantly change the dislocation core structure from that obtained with the local model, but the new formulation leads to significant improvements in the description of dislocation energetics for dislocations with planar cores.

\section{Introduction}

The appropriate method to use in modeling the behaviour of a material is often dictated by some intrinsic length scale in the problem. When considering atomic scale defects, the nanometer length scale is dominant, and fully atomistic models are often required. On the other hand, macroscopic models are concerned with bulk properties of specimens with dimensions on the micron scale or larger. In this regime, the simplifying assumptions of continuum mechanics are justified. Recently, there has been increased interest in modeling the so-called mesoscale regime, the range of length scales that are often too large for fully atomistic models but too small for discrete lattice effects to be ignored. In this regime, neither atomistic modeling nor continuum mechanics is satisfactory, and new models which incorporate features from both approaches seem to be necessary.

One class of model which serves as a bridge between the microscopic and macroscopic approaches is that of cohesive zone models. Using a cohesive zone model allows bulk regions to be handled using conventional continuum mechanics, while atomistic effects are incorpo- 
rated only at certain interfaces where it is deemed that they are important. Models of this type were first proposed by Peierls [1] to describe dislocations, by Barenblatt [2] to model fracture processes and by Dugdale [3], and Bilby, Cottrell and Swinden [4] to estimate plastic zone sizes ahead of cracks. A review of a number of examples of this approach may be found in Miller and Phillips [5], while this paper focuses on the specific example of the Peierls dislocation.

The Peierls dislocation model has received renewed interest of late, being used, for example, by Rice [6] in his description of the brittle versus ductile behaviour of crystals. The attractiveness of the Peierls framework is that it offers an analytically tractable (or at least numerically expedient) continuum model which incorporates nonlinear features resulting from the presence of the discrete lattice. The model assumes that the atomistic features of a dislocation are confined to a single atomic plane referred to as the slip plane. It is only at the slip plane that discontinuities in the displacement fields are assumed to take place, and a special constitutive law is used to account for them. Away from the slip plane, the remainder of the bulk crystal is treated as a linear elastic medium. In conventional treatments, the constitutive law at the slip plane is simplified by assuming that the energy and stress depend only locally on the slip distribution, despite the non-local nature of atomic interactions. In effect, while the Peierls model does incorporate a periodic length into the local relation between stress and slip, it does not define a length scale below which the non-local effects due to slip gradients become important.

In earlier work [5] it has been demonstrated that the failure to incorporate non-local effects is a serious concern, since full atomistic calculations show that the gradients in the slip distribution that are present in realistic dislocation cores are often too large for non-local effects to be ignored. By postulating a new form for the energy of a slip distribution, we formulate a model which includes a non-local term while at the same time reduces to the original Peierls model in the limit where slip gradients become small.

Many examples exist where a certain model, derived under specific assumptions, is pushed to the extremes of its range of applicability and as a result must be corrected through inclusion of higher order terms. Anharmonic models of lattice vibrations [7], [8], recent work on gradient models [9], 10], and several papers by Eringen and co-workers [11]-13] on 
non-local continuum theories are but a few examples of this strategy. Similar extensions are made here in the context of the Peierls framework. Due to the inclusion of a new term in the expression for the energy of a slip distribution, the model is now able to capture non-local effects due to the presence of gradients in the slip distribution. This improvement is demonstrated via comparisons to fully atomistic calculations of slip plane energies, and is then used to model dislocation core structures.

In section 2 we begin with a brief description of the original Peierls framework and its breakdown in the limit of rapidly varying slip distributions. In section 3 , we present the details of our non-local formulation and describe how atomistic calculations are used to build the necessary non-local constitutive model. We then use the non-local Peierls model to compute the energetics of crystalline slip, and show that the non-local model is in better agreement with purely atomistic results than was the classical Peierls framework. Finally, in section 4, the non-local model is used to obtain the core structure of a (100)[011] dislocation in fcc Al.

\section{Breakdown of the Traditional Peierls Framework}

In this section, we present a brief explanation of the Peierls model, but focus mainly on describing the breakdown of the local cohesive zone assumptions. For a complete description of the Peierls model, see Hirth and Lothe [14].

The Peierls model assumes that a dislocation can be described as two elastic half-spaces joined at a common plane on which there is a discontinuous jump in the displacement fields. We adopt the convention that the slip plane is the $x-y$ plane, with the dislocation line along the $y$-axis. The discontinuity in displacements due to the presence of the dislocation is referred to as the slip distribution, $\boldsymbol{\delta}(x, y)=\mathbf{u}^{+}(x, y)-\mathbf{u}^{-}(x, y)$, where $\mathbf{u}^{ \pm}(x, y)$ are the displacement fields just above and below the slip plane. We confine our discussion to the simple case of plane strain in the $x-z$ plane, and to the situation where only one component of $\boldsymbol{\delta}$ is non-zero. This allows us to write $\boldsymbol{\delta}$ as a scalar, $\delta(x)$. Dealing with the more general case is a straightforward extension of the results presented below, while the simple case being discussed here is better suited to demonstrate our arguments. It is important to bear in mind 
that $\delta(x)$ is not constant as in rigid slip, nor is it a simple step function as in the Volterra model of a dislocation. Rather, the slip distribution varies from zero at a point on the slip plane far from the dislocation core to a full Burgers vector once the core is traversed. The slip distribution is assumed to lead to atomic level forces due to the interaction between the slipped surfaces, thus providing the tractions, $\tau(x)$, on the elastic regions. These tractions take a simple form that depends only on the local slip discontinuity

$$
\tau(x)=\tau[\delta(x)]
$$

and can be determined on the basis of atomistic calculations. Early work within the Peierls framework assumed a simple periodic form for eqn. (1), with the periodicity tied to the Burgers vector (see, for example, Foreman, Jaswon and Wood [15]). More recently, highly accurate atomistic calculations have allowed for the direct calculation of $\tau(\delta)$, improving the agreement between models built upon the Peierls framework and the results of direct atomistic simulation (see, for example, Rice, Beltz and Sun [16]).

For the purposes of this paper, we find it more convenient to consider dislocation energetics rather than the resulting forces and tractions. According to the Peierls model, the energy of a dislocation is made up of two parts - the elastic energy contained in bulk regions and the misfit energy associated with the slip plane. The first component, the elastic energy, is fully defined once the elastic constitutive law for the bulk regions is specified, while the misfit energy is computed as an integral over the slip plane. While this is generally a surface integral, for the simplified geometry of a straight dislocation oriented along the y-axis, the integral reduces to

$$
E_{L}=\int_{-\infty}^{\infty} \Phi[\delta(x)] d x
$$

where $E_{L}$ is understood to be the local misfit energy per unit length along the dislocation line. This convention will be maintained throughout the paper. In eqn. (2), $\Phi(\delta)$ is given by

$$
\Phi(\delta)=\int_{0}^{\delta} \tau\left(\delta^{\prime}\right) d \delta^{\prime} .
$$

$\Phi(\delta)$ is referred to as the interplanar slip potential, and can be thought of as the energy cost associated with slipping one block of atoms over another by an amount $\delta$. As with the 
tractions $\tau(\delta)$, the interplanar slip potential can also be obtained through simple atomistic calculations (see, for example, Rice et al. [16] or Kaxiras and Duesbery [17]).

The energetic description provided above introduces an important assumption that has been central to the Peierls framework and results in a formulation that is strictly local. Specifically, this assumption arises from the fact that the interplanar potential is computed on the basis of a purely uniform slip distribution, even though the actual slip distributions of interest are non-uniform. The energy of the non-uniform slip distribution is found using eqn. (2), which effectively divides the slip distribution into infinitesimal slip steps, samples the interplanar potential for each of these steps, and sums the results. The tacit assumption of this approach is that despite the non-uniform nature of the slip distribution, the local environment at each point can be considered to be approximately uniform, allowing for the local slip energy to be determined from the interplanar slip potential. It is expected that as long as the gradients in the slip distribution are small, this approximation will be valid, but as the gradients become more severe, the approximation will breakdown. One result of interest is to quantify what the maximum acceptable gradients are, and to determine whether or not the slip gradients occurring in real dislocation core structures exceed these values.

These questions about the validity of the assumptions in eqn. (2) were addressed by Miller and Phillips [5]. The breakdown of the local assumptions was quantified by computing the energy of a number of idealized slip distributions in two ways, first using atomistics (considered "exact" in this context) and then using the approximation embodied in eqn. (2). The parameters in the slip distribution were then selectively varied, allowing for control of the severity of the slip gradients and a direct comparison of the two methods of obtaining the slip plane energy. These calculations demonstrated a clear breakdown in the locality assumption. Further, it was found that the gradients associated with the slip distributions for simulated core structures are of the same order of magnitude as those for which eqn. (2) failed.

In fig. (1), we reproduce the results of Miller and Phillips for the $\{001\}[110]$ slip system in fcc $\mathrm{Al}$ and include new results for the $\{111\}[110]$ slip system. The figure shows a plot of misfit energy per unit area of the slip plane for various slip distributions. For periodic 
distributions, this is computed by dividing the misfit energy per period by the periodic length. For slip distributions which are not periodic, an effective slip area was used, which is defined as the area over which the slip is greater than $1 \%$ of its maximum value. The following slip distributions were used,

$$
\begin{gathered}
\delta(x)=A \sin \frac{2 \pi x}{w} \\
\delta(x)=A \exp \left[-\frac{1}{2}\left(\frac{x}{w}\right)^{2}\right] .
\end{gathered}
$$

For these slip distributions, $w$ can be varied in order to control the slip gradients. Small $w$ corresponds to large gradients, and hence the regime in which we expect the local approximation to fail. To build these slip distributions for an atomistic calculation, one divides a crystal in two, and imposes a different deformation field in the upper and lower halves. Judicious choice of these deformations leads to the appropriate discontinuity at their common surface, and allows for the computation of the misfit energy due to that discontinuity. This is the exact atomistic energy which is compared to the results of eqn. (2) in fig. (11). Further detail of the atomistic calculation of misfit energy is given in section 3 .

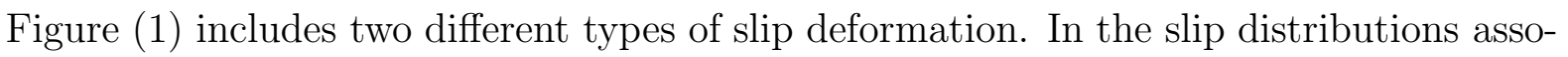
ciated with straight dislocations, two types of gradient effects are possible. First, both the direction in which the slip distribution is changing and the direction of the slip discontinuity itself can be the same. This is characteristic of the slip distributions for pure edge dislocations, and therefore we refer to any slip distribution for which the $\delta$ vector and the $\nabla \delta$ vector are parallel as an "edge"-type distribution. The second type of gradient effect occurs when the direction of the slip discontinuity is perpendicular to the direction along which it is changing. This is characteristic of a pure screw dislocation, and therefore we refer to such an instance as a "screw"-type slip distribution. It is possible to mix these two effects, but for our present purposes we consider only the pure edge and pure screw cases. These two types of slip distributions are analogous to longitudinal and transverse phonons.

The results in fig. (1) demonstrate the breakdown of the Peierls assumption for the slip distributions of eqns. (画) and (5) for both the $\{001\}[110]$ edge distribution and the $\{111\}[110]$ screw distribution. The plots give the energy of the slip distributions as a function of the parameter $w$. In all of the plots, the prediction of the local slip approximation for the 
misfit energy is shown as the constant dashed line near the top of each graph, a striking manifestation of the lack of a characteristic length scale in the local cohesive zone approach. For periodic slip distributions, it is easy to see why the Peierls model approximation is independent of the parameter $w$. The plots of fig. (11) are of the quantity

$$
\frac{E_{L}^{0}}{w}=\frac{1}{w} \int_{0}^{w} \Phi[A \sin (2 \pi x / w)] d x
$$

where $E_{L}^{0}$ is the energy of a single periodic length of the slip plane. The fact that this integral correctly represents the energy of a single periodic length is discussed in section 3 . By making the change of variables $y=x / w$, one can see that the expression is independent of $w$. The data points in fig. (1) come from atomistic calculations of the same misfit energies. Note the divergence from the local estimate at small values of $w$.

The first mission of the non-local model will be to improve the agreement between the atomistic and continuum predictions for the slip energy shown in fig. (11). The solid line in this figure shows the improvements made by using the non-local model, and will be discussed in more detail at the end of section 3 .

\section{The Non-Local Formulation}

The notion that any field variable (for example stress, strain or temperature) is known pointwise, and depends only on other state variables at that point is a natural conclusion of observing the macroscopic behaviour of materials. The result is the assumption of locality one of the basic tenets of the Peierls model, most other cohesive zone models, and classical continuum mechanics in general. However, on the atomic scale the state of each atom is influenced by a finite cluster of its neighbours, not only by the state at an individual atomic site. The non-local formulation of the Peierls model outlined in this section is proposed as a simple way to include the non-local nature of atomic interactions into cohesive zone models such as the Peierls framework.

The modification made to the Peierls model in going from the local to non-local formulation involves the inclusion of a non-local contribution to the misfit energy. The addition 

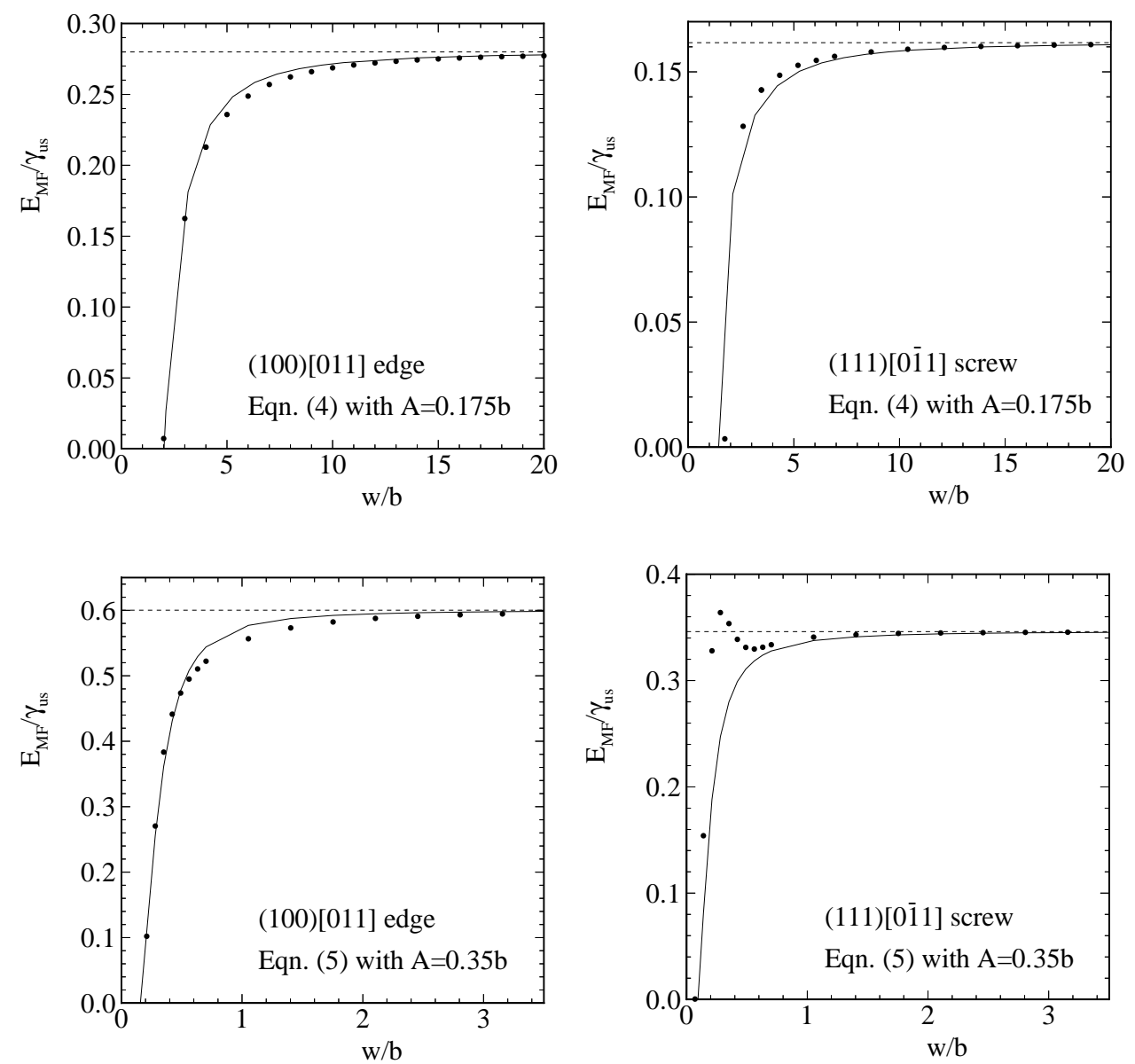

Figure 1: Energy of idealized slip distributions as a function of the parameter $w$, which determines the gradients in the slip distribution. These results demonstrate the failure of the local slip approximation for small $w$. Exact atomistic energy (filled circles); local model (dashed line); non-local model (solid line). 
of this term has far-reaching implications, though it is the only conceptual change that is introduced.

Consider again the misfit energy of a dislocation as given in the local framework of eqn. (2). Non-locality is incorporated by the addition of a term which should vanish for slowly varying slip distributions while at the same time capturing gradient effects when they are present. For a straight dislocation, we postulate the non-local misfit energy to be given by

$$
E_{M F}=\int_{-\infty}^{\infty} \Phi[\delta(x)] d x+\int_{-\infty}^{\infty} \int_{-\infty}^{\infty} K\left(x-x^{\prime}\right) \delta(x) \delta\left(x^{\prime}\right) d x d x^{\prime}
$$

The additional term contains the non-local interplanar kernel $K\left(x-x^{\prime}\right)$, which weights the non-local contributions to the total energy. On physical grounds, we assume that $K\left(x-x^{\prime}\right)=$ $K\left(x^{\prime}-x\right)$, or that the influence that one point has on another depends only on the distance between these points. The new model requires only that we determine a suitable form for the non-local kernel $K\left(x-x^{\prime}\right)$. We propose to adopt a similar strategy to that used in obtaining $\Phi(\delta)$, whereby we extract a numerical reckoning of $K\left(x-x^{\prime}\right)$ from atomistic calculations and then fit these results to simple analytic forms. In the past, non-local expressions of this type have been advanced without the benefit of atomistic calculations to determine the influence function [1]-[13]. It seems possible that the methods presented here can be adapted to those cases as well.

Given the non-local kernel, the traction at the slip plane can be written as the first variation of the misfit energy functional with respect to the slip distribution, yielding

$$
\tau(x)=\frac{\partial E_{M F}}{\partial \delta}=\frac{\partial \Phi[\delta(x)]}{\partial \delta}+2 \int_{-\infty}^{\infty} K\left(x-x^{\prime}\right) \delta\left(x^{\prime}\right) d x^{\prime} .
$$

Thus, for the non-local model, the traction $\tau$ at any point on the slip plane depends on the entire slip distribution, whereas in the local formulation the stress is determined pointwise. It is this additional feature of the new model which allows it to capture the gradient effects discussed earlier.

To determine the non-local kernel, we solve eqn. (7) for $K\left(x-x^{\prime}\right)$ by imposing a number of special slip distributions, and use the non-local term to exactly fit the energies of these slip distributions to the atomistic result. This procedure amounts to the assumption that 
$K\left(x-x^{\prime}\right)$ is independent of the form of the slip distribution. While this may not be rigorously true, it is postulated that the non-local kernel obtained in this way will be reasonably effective for general slip distributions. Based on this assumption, it is convenient to choose slip distributions which allow for a determination of the Fourier components of $K\left(x-x^{\prime}\right)$. For the purposes of our calculation we place two demands on the slip distributions. First, that it be periodic with period $L$, and second, that the maximum amplitude of the distribution is small with respect to the lattice parameter of our material. The first requirement is one of computational convenience, allowing for the use of periodic boundary conditions when determining the exact atomistic energy of a slip distribution, while the second requirement is made in order to allow us to rewrite the interplanar potential, $\Phi$, using its quadratic approximation. This step proves essential in determining $K\left(x-x^{\prime}\right)$ in much the same way that one uses the quadratic approximation to match the shear modulus in the case of the Frenkel sinusoid model. It simplifies the local term in the energy for the purposes of finding the non-local kernel, but once the kernel is computed the exact form of the local term will be reinstated. This ensures that the non-local model will still be applicable to large slip deformations.

The energy expression of eqn. (7) yields the energy of the slip distribution over the entire slip plane. On the other hand, atomistic models naturally provide us with a way to compute the energy of only a finite section of the slip plane. If periodic boundary conditions are used in the atomistic model, as will be used here, then the energy obtained from atomistics is the energy of a single periodic length of the slip distribution. In order to make valid comparisons between the exact atomistic misfit energy and the energy obtained from the non-local model, we must find the non-local expression for the energy of a single periodic length of slip. Equation (7) can be re-written in the form

$$
E_{M F}(\delta)=\sum_{n=-\infty}^{\infty}\left[\int_{n L+\xi}^{(n+1) L+\xi} c \delta(x)^{2} d x+\int_{n L+\xi}^{(n+1) L+\xi} \int_{-\infty}^{\infty} K\left(x-x^{\prime}\right) \delta(x) \delta\left(x^{\prime}\right) d x^{\prime} d x\right],
$$

where the constant $c$ arises from the treatment of the interplanar potential via its quadratic approximation and $\xi$ is some origin where $0 \leq \xi \leq L$. It is easy to show that for periodic slip distributions, the integrals inside this sum are independent of $n$ and $\xi$, and hence each periodic length in the variable $x$ contributes the same amount to the total misfit energy. We 
can then write the misfit energy of a single period of the slip plane, $E_{M F}^{0}(\delta)$, as

$$
E_{M F}^{0}(\delta)=\int_{0}^{L} c \delta(x)^{2} d x+\int_{0}^{L} \int_{-\infty}^{\infty} K\left(x-x^{\prime}\right) \delta(x) \delta\left(x^{\prime}\right) d x^{\prime} d x
$$

and note that for a given slip distribution, $E_{M F}^{0}(\delta)$ is a quantity which can be computed directly from atomistics. For this purpose, it is convenient to take $\delta(x)$ to be

$$
\delta(x)=\delta_{q}(x)=A \sin q x
$$

where $q=2 \pi / L$ and $\mathrm{A}$ is much smaller than the lattice constant of the crystal under consideration. We insert this form of $\delta(x)$ into the expression for $E_{M F}^{0}$. Making a change of variables $z=x-x^{\prime}$ and recalling that $K(z)=K(-z)$, the energy expression becomes

$$
E_{M F}^{0}\left(\delta_{q}\right)=\frac{c A^{2} L}{2}+A^{2} \int_{0}^{L} \sin q x\left[\int_{-\infty}^{\infty} K(z) \frac{e^{i q(z+x)}-e^{-i q(z+x)}}{2 i} d z\right] d x,
$$

where we have made use of the exponential form of the sine function. We recall the definition of the Fourier transform, $\hat{f}(k)$, of a function $f(x)$ as

$$
\hat{f}(k)=\int_{-\infty}^{\infty} f(x) e^{-i k x} d x
$$

and also the inverse Fourier transform

$$
f(x)=\frac{1}{2 \pi} \int_{-\infty}^{\infty} \hat{f}(k) e^{i k x} d k
$$

and use eqn. (13) to write the non-local energy expression in terms of the Fourier transform of the non-local kernel, $\hat{K}(q)$. Noting again that $K(z)=K(-z)$, we see that $\hat{K}(q)=\hat{K}(-q)$, and the expression for the total energy becomes

$$
E_{M F}^{0}\left(\delta_{q}\right)=\frac{c A^{2} L}{2}+A^{2} \hat{K}(q) \int_{0}^{L} \sin ^{2} q x d x .
$$

Evaluating the integral leads to an explicit expression for the non-local interplanar kernel in Fourier space;

$$
\hat{K}(q)=\frac{2 E_{M F}^{0}\left(\delta_{q}\right)}{A^{2} L}-c .
$$

At this point, the strategy is to obtain the energy dependence on the Fourier variable $q$ numerically through an atomistic model. 


\subsection{Atomistic Determination of the Non-Local Interplanar Kernel}

The procedure for determining the energy of a given slip distribution was outlined in section 2 and is described in detail by Miller and Phillips [5]. Here, we use the same computational approach which is described briefly below.

We desire the energy of the slip distribution given by eqn. (11) as a function of the parameter $q$. To make the computation, the crystal is divided into an upper and a lower half. By imposing the appropriate displacement fields on the two half spaces, we can create the desired slip distribution at their common interface. For example, consider the slip distribution of eqn. (11). For the case of edge-type slip, the slip distribution can be obtained by imposing the following displacement fields on the crystal:

$$
\begin{aligned}
\mathbf{u}^{+} & =[(A / 2) \sin q x, 0,0], \\
\mathbf{u}^{-} & =[(-A / 2) \sin q x, 0,0],
\end{aligned}
$$

where $\mathbf{u}^{+}$represents the displacement field in the upper half of the crystal and $\mathbf{u}^{-}$is that in the lower half. This combination of displacement fields leads to a slip distribution of the form

$$
\delta(x)=u_{x}^{+}-u_{x}^{-}=A \sin q x
$$

at the plane where the upper and lower half spaces meet.

Using any convenient atomistic model (we have used the embedded atom method (EAM), see [18] for example.) it is then possible to compute the energy of this crystal. The result of such an atomistic calculation is an energy consisting of two parts, that due to the interface and an additional elastic strain energy due to the deformation in the bulk regions of the crystal. The elastic energy can be found directly by computing the energy of each half space separately, using a periodic computational cell containing no slip discontinuities. This energy can then be subtracted from the total energy of the configuration which includes the slip jump, leaving the misfit energy, $E_{M F}^{0}$.

By repeating this procedure for a sequence of values of $q$, we obtain a discrete represen-

tation of the non-local kernel $\hat{K}(q)$ in Fourier space. Results of such calculations using the EAM potentials for $\mathrm{Al}$ of Ercolessi and Adams [19] are presented in fig. (2). The various slip 
systems are either edge, screw or "mixed", where edge and screw are as defined in section 2. The "mixed" system is in this case the direction associated with the 30 degree Shockley partial. All of the curves are given in non-dimensional form using the constants given in table (回), where $\gamma_{u s}$ is the unstable stacking fault energy for a given slip system. The values in the table are obtained by rigidly sliding two blocks of atoms with respect to one another and allowing relaxations in only the out-of-plane direction. The remaining constants in the table are $b$, the Burgers vector, and $d$, which is defined below. The representative examples in fig. (21) demonstrate a number of the characteristics of the non-local interplanar kernel when constructed in Fourier space. All such functions are even and periodic, and therefore only the first half period of each is shown. The periodic length of $\hat{K}$ depends on the slip system being considered, and is given by $2 \pi / d$ where $d$ is the distance between planes with normal parallel to the direction of the slip gradient vector. Note that these planes are a set of $\mathrm{y}-\mathrm{z}$ planes, which are perpendicular to the $\mathrm{x}-\mathrm{y}$ slip plane. Another characteristic feature of the $\hat{K}$ function is that it is exactly zero at $q=0$, which implies that in the limit of very slowly varying slip distributions, the non-local correction vanishes and we recover the classical Peierls framework. It is also worth noting that while most of the $\hat{K}$ functions are negative, we see that for the case of screw-type slip distributions on the $\{001\}[010]$ slip system $\hat{K}$ is entirely positive. This suggests that there is no set rule about whether the non-local contribution to the misfit energy is positive or negative - it will depend on both the slip system being considered and on the Fourier components of the slip distribution itself. Comparing the various curves in this figure can help us to understand the relative importance of the non-local effect for various slip systems. Note that the strongest effect is associated with the (100)[010] edge system. It is interesting that this same slip system, but in the screw orientation, shows the weakest non-local correction, and that the sign of this correction for the various Fourier components of the slip distribution is reversed. This is because the screw-type sinusoidal slip distribution represents a more severe misfit configuration than the edge-type for this slip system. Meanwhile, the local model predicts the same energy for either edge or screw slip due to the symmetries of the $\{100\}$ planes. For this slip system, the local prediction for misfit energy somewhat overestimates the edge energy, while somewhat underestimating its screw counterpart. The result is this seemingly anomalous behaviour of the screw-type slip. 


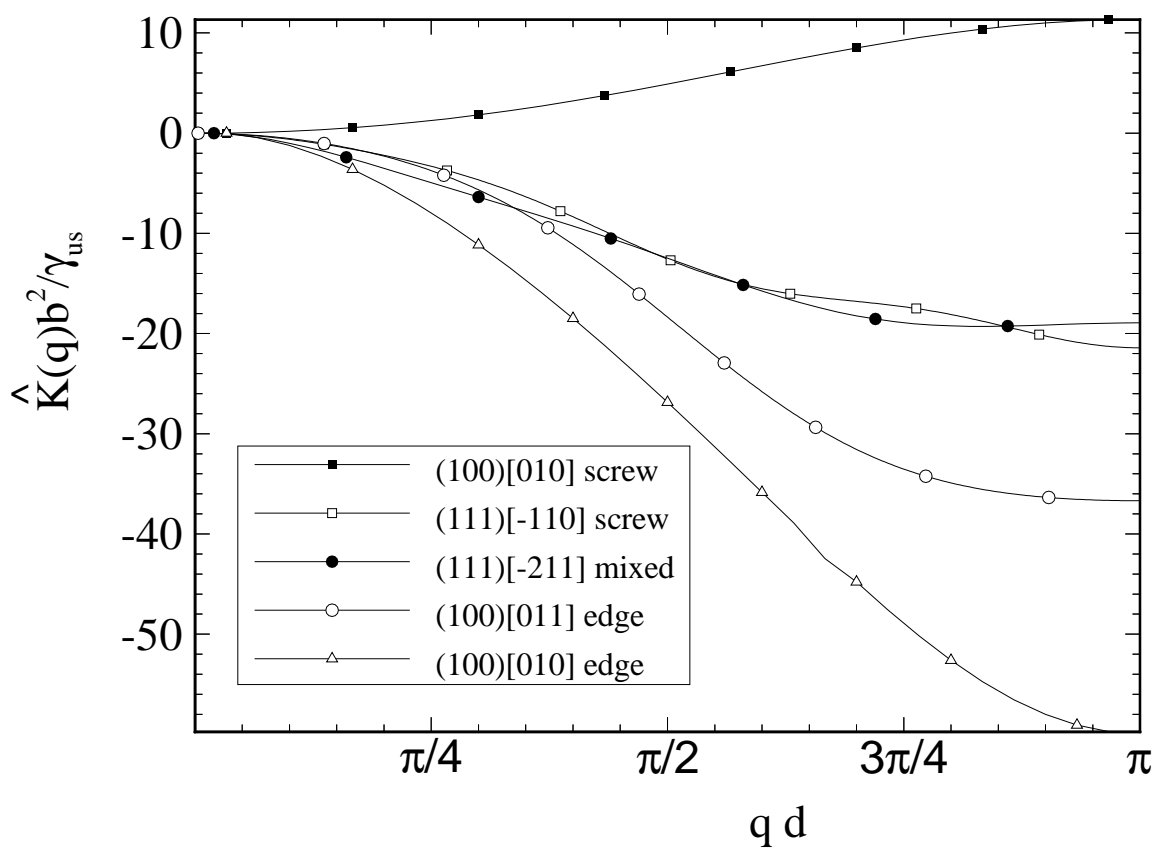

Figure 2: Examples of the Fourier space representation of the non-local interplanar kernel, $\hat{K}$, for various slip systems in $\mathrm{Al}$, as obtained using the EAM potentials of Ercolessi and Adams [19]. See table (1) for normalization constants.

\begin{tabular}{|c|c|c|c|}
\hline Slip System & $b(\AA)$ & $d / b$ & $\gamma_{u s}\left(e V / \AA^{2}\right)$ \\
\hline$(100)[010]$ screw & 4.032 & $1 / 2$ & 0.04065 \\
$(111)[\overline{1} 10]$ screw & 2.851 & $\sqrt{3} / 6$ & 0.02705 \\
$(111)[\overline{2} 11]$ mixed & 1.646 & $\sqrt{3} / 2$ & 0.008032 \\
$(100)[011]$ edge & 2.851 & $1 / 2$ & 0.02521 \\
$(100)[010]$ edge & 4.032 & $1 / 2$ & 0.04065 \\
\hline
\end{tabular}

Table 1: Burgers vector $b$, interplanar spacing $d$ and unstable stacking fault energy $\gamma_{u s}$ for the slip systems considered in fig. (2) as obtained from the EAM Al potentials of Ercolessi and Adams [19]. 


\subsection{Approximate Analytic Form in Real Space}

Numerical inversion of the Fourier representation of $\hat{K}$ deduced above can be problematic due to the fact that $\hat{K}$ is known only for a discrete set of points in Fourier space. Therefore, we propose to fit $\hat{K}$ in Fourier space with a cosine series, which can be easily transformed into real space in the form of a sum of Dirac delta functions. Because $K\left(x-x^{\prime}\right)$ exists as part of an integrand over the entire slip plane, these Dirac deltas will have the effect of reducing the dimension of the integral in eqn. (7). We have found that excellent fits of the numerical data can be obtained using only the first few terms of such a cosine series, and one can therefore write

$$
\hat{K}(q) \cong \frac{a_{0}}{2}+\sum_{n=1}^{N} a_{n} \cos n d q
$$

where $a_{n}$ are the fitting parameters and $d$ is the distance between planes perpendicular to the slip plane, as described previously. The number of fitting parameters $N$ will depend on the range of the atomistic potentials used in obtaining $\hat{K}$, but we have found that for the potentials and slip systems considered here, the value of $N$ typically does not need to exceed five. This form for the non-local kernel allows for easy Fourier inversion, yielding

$$
K\left(x-x^{\prime}\right)=\frac{a_{0}}{2} \delta_{D}\left(x-x^{\prime}\right)+\sum_{n=1}^{N} \frac{a_{n}}{2}\left(\delta_{D}\left(x-x^{\prime}+n d\right)+\delta_{D}\left(x-x^{\prime}-n d\right)\right),
$$

where $\delta_{D}$ is the Dirac delta function. This expression for the non-local kernel can be used in the original definition for the misfit energy, as well as in the expression for the tractions on the slip plane. The results are

$$
\begin{gathered}
E_{M F}=\int_{-\infty}^{\infty} \Phi[\delta(x)] d x-\sum_{n=1}^{N} \int_{-\infty}^{\infty} a_{n}\left(\delta(x)^{2}-\frac{\delta(x) \delta(x-n d)+\delta(x) \delta(x+n d)}{2}\right) d x \\
\tau(x)=\frac{\partial \Phi[\delta(x)]}{\partial \delta}-\sum_{n=1}^{N} a_{n}(2 \delta(x)-\delta(x+n d)-\delta(x-n d))
\end{gathered}
$$

where we have eliminated $a_{0}$ from the expressions by using the fact that $\hat{K}(0)=0$. The $\delta$ appearing in this expression is the slip distribution, and not the Dirac delta function $\delta_{D}$. The computed values of $a_{n}$ are plotted in fig. (3) for the five slip systems considered here. In this figure, increasing $n$ corresponds to sampling the slip distribution farther and farther 

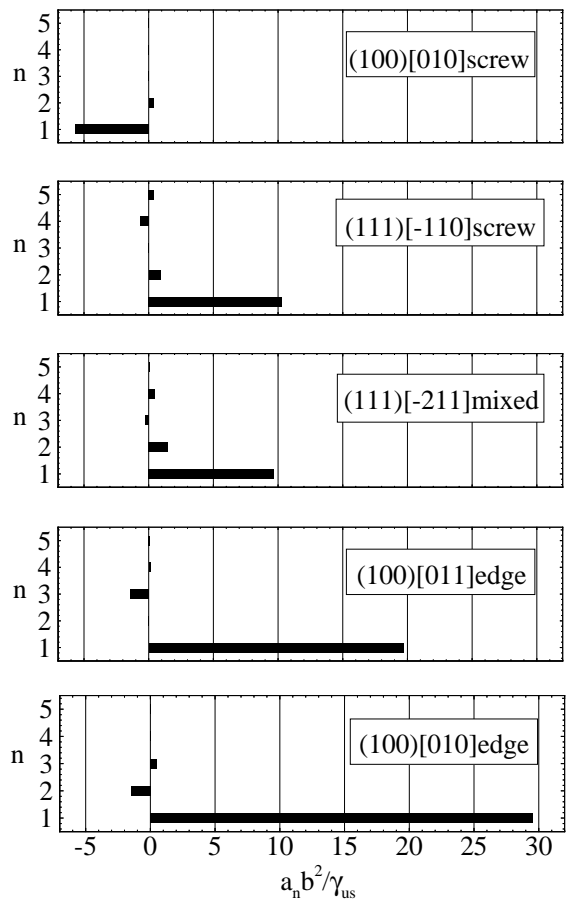

Figure 3: Non-local influence coefficients, $a_{n}$, for the slip systems considered in fig. (2).

from the point at which the energy and stress is being computed. It is clear that the nonlocal effects decays quite rapidly, and in all cases $a_{1}$ (corresponding to the near neighbour non-local influence) is by far the most important contribution. Non-local influence is felt as far as five neighbours away in some systems, but beyond $a_{5}$, the coefficients are negligible.

Note that the expressions for the energy and stress are consistent with our intuition as to the nature of the non-local terms. First, in the limit when $\delta(x)$ is slowly varying, $\delta(x \pm n d) \rightarrow \delta(x)$ and the above expressions reduce to the conventional local formulation. Second, the non-local effects for a given point arise as a result of sampling a discrete set of points along the rest of the slip distribution. These points are spaced by a distance $d$, which coincides with the spacing of atomic planes in the direction of the slip gradient vector. The discreteness of this sampling, together with the physical significance of the spacing of the sampling points results in a sensible atomistic extension of the original constitutive assumptions of the local model.

Finally, it is interesting to note that the terms of the sum in eqn. (23) can be viewed 
as a linear combination of approximations to the derivatives of the slip distribution. The symmetry of our non-local kernel in Fourier space means that only even order derivatives contribute to this correction, and therefore it is possible to re-write eqn. (23) in the form

$$
\tau(x)=\frac{\partial \Phi[\delta(x)]}{\partial \delta}-\sum_{n=1}^{N} c_{2 n} \delta^{(2 n)}(x),
$$

where the coefficients $c_{n}$ are the weights associated with the $n$th derivative, and $\delta^{(n)}$ is the central difference approximation to the $n$th derivative of the slip distribution. The $c_{n}$ are simply linear combinations of the $a_{n}$ already introduced. For example, if we take $N=2$ in eqns. (23) and (24), and make use of the following central difference approximations for the second and fourth derivative of $\delta(x)$,

$$
\begin{gathered}
\frac{d^{2} \delta}{d x^{2}}=\frac{\delta(x+d)-2 \delta(x)+\delta(x-d)}{d^{2}} \\
\frac{d^{4} \delta}{d x^{4}}=\frac{\delta(x+2 d)-4 \delta(x+d)+6 \delta(x)-4 \delta(x-d)+\delta(x-2 d)}{d^{4}}
\end{gathered}
$$

then we can solve for the coefficients $c_{2}$ and $c_{4}$. In this case, these are found to be

$$
\begin{gathered}
c_{2}=-d^{2}\left(a_{1}+a_{2}\right), \\
c_{4}=-d^{4} a_{2} .
\end{gathered}
$$

This exercise highlights the parallels between our non-local approach and recent gradient correction models (e.g.[9], [10]), although in this paper we will not explore the gradient correction form of these equations further.

\subsection{Energy of Crystalline Slip Within the Non-Local Formulation.}

The simplest test of the non-local model is to re-examine the energetics of idealized slip distributions as originally presented in fig. (1). Recall that in this figure the predictions of the local model are given by the constant dashed line near the top of each graph, whereas the data points are exact calculations of the misfit energy as determined using atomistics. The solid curves are the results of using the non-local model, which are in significantly better agreement with the exact results than are the local results. In each case, the energetics 
using the non-local model exhibit the correct trend as a function of slip gradients, unlike the original model which does not capture gradient effects. The non-local model is not able to capture the anomalous upturn in the atomistic results for the $\{111\}[110]$ screw orientation, a pathology of this particular slip deformation which results from putting atoms into highly unfavourable proximity across the slip plane. Nonetheless from the standpoint of purely energetic considerations, the non-local model shows a marked improvement over the local model, without any great cost in model complexity.

Emboldened by our observations that the non-local correction improves the energetic description of crystalline slip, we now proceed to a result of greater interest, namely, the effect of the non-local terms in the context of realistic dislocation core structures.

\section{The Non-local Model of Realistic Dislocation Cores}

As an example, we apply the non-local model to the determination of the core structure for a straight dislocation with a planar core. For this purpose we consider the Lomer dislocation in fcc Al, with a $<110>$ line direction and Burgers vector $\frac{a}{2}<\overline{1} 10>$.

We obtain the Lomer core structure using two schemes. The first makes use of a simplified form for the interplanar potential $\Phi(\delta)$ that allows for a closed form analytic solution for the slip distribution in Fourier space. In the second determination of the Lomer core, the full-blown atomistic result for $\Phi(\delta)$ is used and the core structure is computed numerically. Each of these results is then compared to the slip distribution taken directly from the atomic positions resulting from full relaxation of the atomistic degrees of freedom. The first scheme, which admits of an analytic solution, is of interest as a method for testing the numerical procedures used in the second scheme. At the same time, it demonstrates that considerable analytical progress can be made with the non-local formulation. The second, fully numerical solution demonstrates that stable core structures are readily obtainable within the non-local formulation. We will see that while the effect of the non-local terms on the slip distribution and core structure are subtle to the eye, they represent a significant improvement when quantified in terms of the predicted misfit energy of the dislocation core. 


\subsection{Eigenstrains solution for $\delta$ within the quadratic well approx- imation.}

In this section, we describe the procedure used in determining an analytic result for the core structure of the Lomer dislocation. The important approximation in this procedure in relation to the results of the subsequent section is the simplified form used for the interplanar potential $\Phi(\delta)$.

Within the Peierls framework, the determination of $\delta(x)$ is equivalent to finding the core structure of the dislocation. Once $\delta(x)$ is found, the elastic displacement fields away from the slip plane can be computed from an integral of the Volterra kernel over the entire slip plane. As well, the misfit and elastic energy are then fully specified by the slip distribution. Therefore, we seek the slip distribution $\delta(x)$ which minimizes the total energy functional. The energy associated with the dislocation, which is a functional of the slip distribution $\delta(x)$, can be expressed as the sum of three parts

$$
E_{t o t}=E_{L}+E_{N L}+E_{B}
$$

$E_{L}$ is the misfit energy as determined within the traditional local model and given in eqn. (22), $E_{N L}$ is the non-local correction term added to the misfit energy in eqn. (7), and $E_{B}$ is the elastic energy of the bulk region. The elastic term is obtained by superimposing the elastic interaction energy for a distribution of infinitesimal dislocations with Burgers vector density $-d \delta / d x$

$$
E_{B}=\frac{1}{2} \int_{-\infty}^{\infty} \int_{-\infty}^{\infty} B \log \left(\frac{R}{\left|x-x^{\prime}\right|}\right) \frac{d \delta(x)}{d x} \frac{d \delta\left(x^{\prime}\right)}{d x^{\prime}} d x d x^{\prime}
$$

In this equation, $R$ is a measure of the size of the bulk region. Making use of integration by parts, it is possible to eliminate the constant $R$ from the analysis. $B$ is defined as

$$
B=2 C_{i j} s_{i} s_{j}
$$

where $s_{i}$ is the $i$ th component of the slip direction for the dislocation of interest and $C_{i j}$ is the prelogarithmic energy tensor, discussed in detail, for example, in Bacon, Barnett and Scattergood [20]. In the case of an isotropic solid $B$ reduces to

$$
B_{i s o}=\frac{\mu}{2 \pi(1-\nu)}
$$




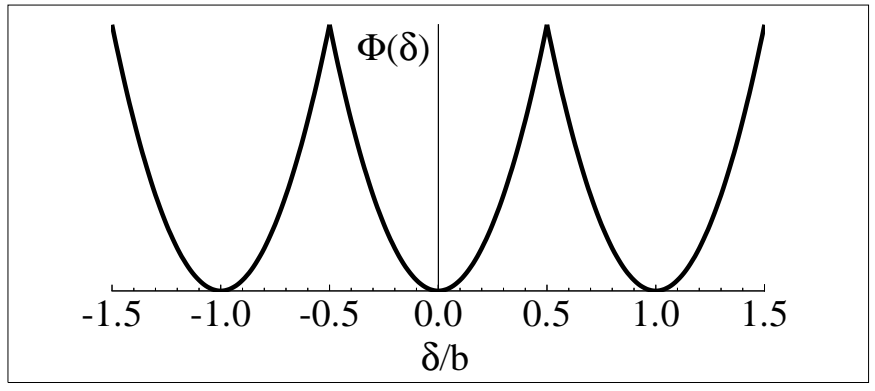

(a)

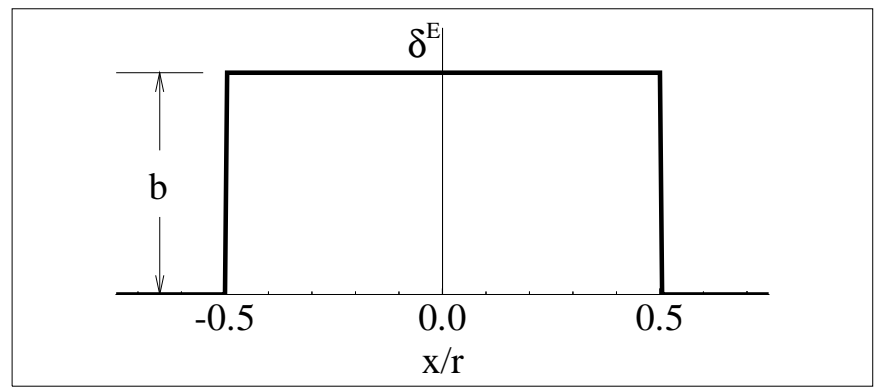

(b)

Figure 4: (a) Quadratic well approximation to the local interplanar potential. (b) Eigenslip, $\delta^{E}(x)$ for the dipole configuration. 
For the purposes of obtaining an analytic solution, we make the approximation that the local interplanar potential $\Phi(\delta)$ takes the form of a periodic array of quadratic wells as shown in figure (4a). We then employ the method of eigenstrains as described by Mura [21] to minimize the energy functional with respect to the slip distribution for a dislocation dipole with spacing $r$. The dipole configuration is used so that the slip distribution goes to zero at $x= \pm \infty$. By making $r$ large, the dipole becomes two isolated dislocations of opposite sign. This approach allows us to rewrite the local contribution to the energy as

$$
E_{L}=\int_{-\infty}^{\infty} c\left(\delta(x)-\delta^{E}(x)\right)^{2} d x
$$

In this equation the constant $c$ is determined by fitting the quadratic wells to linear elasticity at small values of $\delta-\delta^{E}$. This leads to $c=\mu / 2 a$ where $a$ is the spacing between slip planes and $\mu$ is the relevant shear modulus for the slip system of interest. $\delta^{E}$ in this expression is the "eigenslip" which ensures that the dipole slip configuration is enforced during the energy minimization. $\delta^{E}(x)$ is shown in figure (बb), where $b$ is the Burgers vector and $r$ is the dipole spacing. We will need the Fourier transform of this eigenslip, which is found to be

$$
\hat{\delta}^{E}=\int_{-r / 2}^{r / 2} b e^{-i k x} d x=\frac{2 b}{k} \sin \frac{k r}{2} .
$$

Making use of eqn. (14), we can replace $\delta, \delta^{E}$ and $K$ with their Fourier transforms, allowing for a Fourier space representation of the total energy. Integrating by parts on the bulk energy terms and assuming that the order of integrations can be switched allows us to simplify the energy expression to

$$
E_{t o t}=\int_{-\infty}^{\infty}\left[\hat{\delta}(k) \hat{\delta}(-k)\left(\frac{c}{2 \pi}+\frac{\hat{K}(k)}{2 \pi}+\frac{B}{4}|k|\right)-\frac{c}{\pi} \hat{\delta}(k) \hat{\delta}^{E}(-k)+\frac{c}{2 \pi} \hat{\delta}^{E}(k) \hat{\delta}^{E}(-k)\right] d k
$$

Note that at this point, our solution is predicated upon a knowledge of the Fourier space features of the interplanar kernel. Taking variations with respect to $\hat{\delta}$ and setting $\delta E_{\text {tot }}=0$ we find

$$
\hat{\delta}(k)=\frac{2 b \sin \frac{k r}{2}}{k\left[1+\frac{\hat{K}(k)}{c}+\frac{\pi B}{2 c}|k|\right]}
$$

where we have made use of the fact that $\hat{K}(k)$ is an even function and used eqn. (34) to replace the Fourier transform of the eigenslip. This expression gives us the Fourier transform 
of the slip distribution for the dislocation dipole. Although it cannot be transformed into real space explicitly, the transformation integral is straightforward to perform numerically once the form of $\hat{K}$ is known. Thus we numerically compute the slip distribution from the expression

$$
\delta(x)=\frac{2 b}{\pi} \int_{0}^{\infty} \frac{\sin \frac{k r}{2} \cos k x}{k\left[1+\frac{\hat{K}(k)}{c}+\frac{\pi B k}{2 c}\right]} d k .
$$

This result is plotted in fig. (5) for the case of $\hat{K}=0$ (which corresponds to the local Peierls model solution) and with $\hat{K}$ from fig. (2) for the (100)[011] edge (Lomer) slip system. Note that only one member of the dipole pair is shown because of the symmetry of the distribution. We see in fig. (5a) that the effect of the non-local correction is to introduce small oscillations in the slip distribution and increase its slope slightly at the dislocation core. At first, the oscillations may appear to be unphysical, but recall that this continuous curve is really a representation of the slip for a discrete set of lattice sites. Therefore, only the values at the lattice sites are germane for the atomic positions implied by the solutions. It is interesting to note that this analytic model provides a reasonable approximation to the dislocation core, although the differences between the local and non-local results are hard to quantify. We should not expect the continuum model to accurately capture subtle details of the atomic core, but it is encouraging that the model does lead to a stable core configuration that is similar to the exact result.

\subsection{Numerical solution for the slip distribution.}

It is now of interest to see what effect the non-local correction has on the dislocation core structure when the correct atomistically obtained $\Phi(\delta)$ is used. In this case, it is necessary to resort to a full numerical solution of the governing equation.

The numerical approach for finding $\delta(x)$ follows closely the approach of Beltz and Freund [22], to which we refer the interested reader for more details. We begin with the well known Peierls-Nabarro equation [14]

$$
\tau[\delta(x)]=-\frac{\mu}{2 \pi(1-\nu)} \int_{-\infty}^{\infty} \frac{d \delta\left(x^{\prime}\right) / d x^{\prime}}{x-x^{\prime}} d x^{\prime}
$$

into which we can substitute eqn. (23) for $\tau[\delta(x)]$. By an appropriate change of variables, 

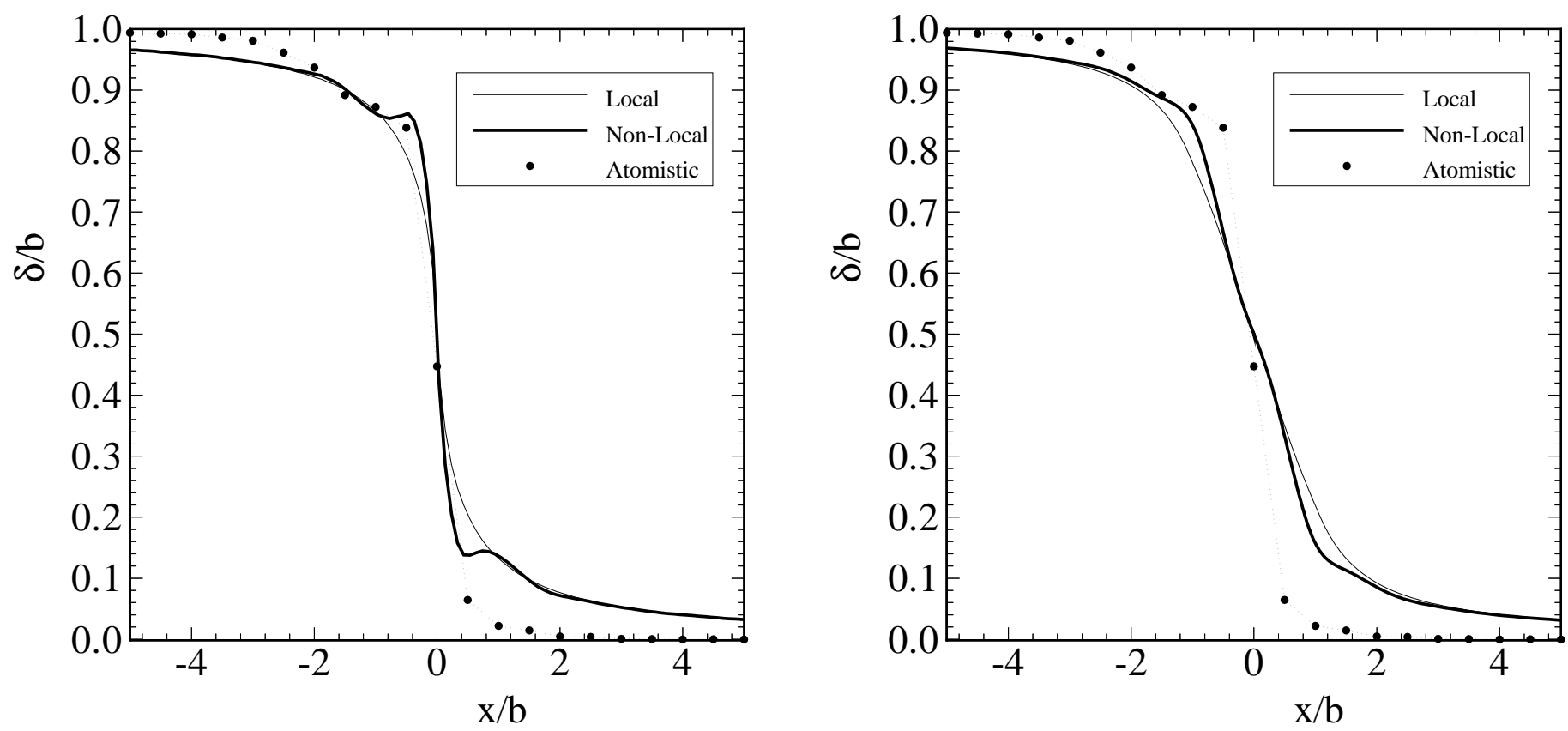

Figure 5: Comparison of core structures for the Lomer dislocation in $\mathrm{Al}$ as obtained from the continuum model with exact atomistic results. (a) Structure obtained using the quadratic well model of the interplanar potential and (b) with the full numerical solution to the PeierlsNabarro equation. 
the domain of integration can be collapsed onto the finite domain $(-1,1)$. This domain can be discretized and the integration carried out by making use of the Gauss-Chebyshev integration techniques described in Erdogan and Gupta [23]. This approach reduces the problem to a set of nonlinear algebraic equations which can be solved iteratively via the Newton-Raphson method, leading to a discrete representation of the slip distribution $\delta(x)$. The results of such a calculation for the Lomer core structure are presented in fig. (5b). Again, we note that the resulting core structure is not changed dramatically relative to the local model as a result of adding the non-local correction. Neither the local nor the non-local model can fully capture the details of the core, and it is difficult, on the basis of visual inspection, to quantify the virtues of including the non-local correction when considering only the slip distributions.

The fact that the structural differences between the local and non-local models are small does not imply that the non-local effect is unimportant. Instead, the important quantity to compare between the two models is their predictions for the total energy of the dislocation. The way the elastic energy is computed for the two models is identical, and the highly similar predicted cores mean that the value of the elastic energy for the two models will be about the same. On the other hand, the misfit energy is computed differently, and even identical core structures will lead to different misfit energies by virtue of the non-local energy term.

Using the method described in Miller and Phillips [5] to isolate the misfit energy from the strain energy in the bulk regions, we can compute the exact atomistic misfit energy of the lomer dislocation to be $0.1362 \mathrm{eV} / \AA^{2}$. This is accomplished by representing the atoms in the upper and lower bulk regions by nodes in a finite element mesh, and computing the strains (and consequent strain energy) in this mesh via the Cauchy-Born rule [24]. On the other hand, we can compute this energy using the local and non-local models from eqns. (2) and (22) respectively. The local model predicts an energy of $0.1979 \mathrm{eV} / \stackrel{\circ}{ }^{2}$, about $45 \%$ greater than the exact energy. The non-local result is $0.1471 \mathrm{eV} / \AA^{2}$, only $8 \%$ greater than the atomistic result. This result demonstrates that although the core structures in the local and non-local formulations are for practical purposes indistinguishable, the non-local treatment of the core energies is significantly more accurate. We would expect similar adjustments to the cohesive zone model estimate of the Peierls stress. 


\section{Conclusions}

Motivated by previous work which demonstrated a failure of the local Peierls framework to accurately describe the energy of interplanar slip for the types of slip distributions found in real dislocation cores, we proposed a non-local formulation of this framework. We then proceeded to outline a set of simple atomistic calculations whereby one can obtain the non-local interplanar kernel required in the formulation of the model. To demonstrate that the model improved estimates of the slip energy, we compared these results to purely atomistic calculations and showed that the non-local model improves the agreement between the cohesive zone model energies and explicit atomistic energies.

Given that the non-local model leads to better energetic descriptions of slip distributions, we proceeded to demonstrate the model by computing the structure of a straight dislocation with a planar core, namely, the Lomer dislocation in Al. It was found that the differences between the local and non-local results, and between either of them and the atomistic core, were subtle and difficult to quantify when considering only the spatial structure of the dislocation. However, the important quantitative measure of the performance of the models is the energy, which the local model predicted to be $45 \%$ larger than the exact atomistic result. The non-local model significantly improved the energetic description of the core, overestimating the exact result by only $8 \%$. Nevertheless, the negligible changes in the nonlocal description of the core structure serve as a reminder that the cohesive zone approach appears to lack the flexibility to really serve as a generic basis for mixed atomistic and continuum studies of dislocations. Even in its non-local form, this framework restricts the slip to a particular slip plane, thus forbidding the emergence of complex cores such as those found in bcc metals.

Future work in the context of cohesive zone models could include an attempt to integrate non-local effects into other models of the mechanical behaviour of materials. One example of interest is the model of Rice [6] and the numerous related works that describe dislocation emission from crack tips. Preliminary investigations in this area suggest that the concepts outlined here can be used for such problems [25]. The basic equation to be solved in the case of a dislocation near a crack tip is a modified version of the Peierls-Nabarro equation with 
additional terms due to the presence of the crack. Any realistic solution of this equation requires a numerical procedure, and the simple form of the non-local corrections in eqns. (22) and (23) mean that the additional computational cost in the non-local formulation is small. The most serious obstacle in this case seems to be the proper treatment of the boundary conditions in the non-local setting, and most importantly the stress free boundaries at the crack faces. However, the fact that non-locality plays an important role at the atomic level means that a correct non-local treatment of the atomistically sharp crack may be an important contribution to our understanding of crack tip phenomena.

Another possible direction for this work is the integration of the non-local formulation into the recently proposed "semi-discrete Peierls framework" of Bulatov [26]. Results using this version of the Peierls model show significant improvement to the conventional Peierls framework, but some error remains. It is possible that including non-local effects into this model may further improve its agreement with atomistics while retaining its tractability.

A third future direction for this research should be an effort to compute other non-local kernels analogous to $K\left(x-x^{\prime}\right)$. For example, it may be possible to directly compute from atomistics the non-local elastic moduli introduced by Eringen and co-workers [11]-[13] for bulk crystals. This would eliminate guesswork about their appropriate form and base them solidly on their atomistic underpinnings. Other recent work ([27], 28]) substantiates our belief in the critical role played by constitutive non-locality in the description of atomic scale defects and calls for continued efforts to put such models on a clear analytic footing.

\section{Acknowledgements}

This work was supported by the Natural Sciences and Engineering Research Council of Canada, by NSF grants CMS-9414648 and CMS-9502020 and by the National Science Foundation under the Materials Research Group grant No. DMR-9223683. We are grateful to S. Foiles and M. Daw for the use of their code Dynamo. Finally, it is a pleasure to acknowledge useful conversations with V. Bulatov, A. Carlsson, M.S. Duesbery, J.R. Rice, V. Shenoy and E. Tadmor. 


\section{References}

1. Peierls, R.E., 1940, Proc. Phys. Soc. Lond., 52, 34.

2. Barenblatt, G.I., 1962, Adv. Appl. Mech. 7, 55.

3. Dugdale, D.S., 1959, J. Mech. Phys. Sol., 8, 100.

4. Bilby, B.A., A.H. Cottrell and K.H. Swinden, 1962, Proc. Roy. Soc. Lond., A272, 304.

5. Miller, R. and R. Phillips, 1996, Phil. Mag. A, 73, 803.

6. Rice, J.R., 1992, J. Mech. Phys. Sol., 40, 239.

7. Duesbery, M.S., R. Taylor and H.R. Glyde, 1973, Phys. Rev. B, 8, 1372.

8. DiVincenzo, D.P., 1986, Phys. Rev. B., 34, 5450.

9. Fleck, N.A., G.M. Muller, M.F. Ashby and J.W.Hutchinson, 1994, Acta Met. et Mat., 42,475 .

10. Aifantis, E.C., 1992, Int. J. Engng. Sci., 30, 1279.

11. Eringen, A. C., C.G. Speziale and B.S. Kim, 1977, J. Mech. Phys. Sol., 25, 339.

12. Eringen, A. C. and F. Balta, 1979, Crystal Lattice Defects, 8, 73.

13. Eringen, A. C., 1987, Res. Mech., 21, 313.

14. Hirth, J.P., and J. Lothe, 1992, Theory of Dislocations (Malabar, Florida: Krieger).

15. Foreman, A.J., M.A. Jaswon and J.K. Wood, 1951, Proc. Phys. Soc. Lond. A, 64, 156.

16. Sun, Y., G. E. Beltz and J. R. Rice, 1993, Mat. Sci. Eng. A, 170, 67.

17. Kaxiras, E. and M. S. Duesbery, 1993, Phys. Rev. Lett., 70, 3752.

18. Daw, M.S. and M. I. Baskes, 1984, Phys. Rev. B, 29, 6443.

19. Ercolessi, F. and J. B. Adams, 1994, Europhys. Lett. 26, 583.

20. Bacon, D.J., D.M. Barnett and R.O. Scattergood, 1979, Prog. Mat. Sci., 23, 51. 
21. Mura, T., 1984, Micromechanics of Defects in Solids, 2nd Ed., (The Netherlands: Kluwer Academic).

22. Beltz, G.E., and L.B. Freund, 1994, Phil. Mag. A, 69, 183.

23. Erdogan, F., and G.D. Gupta, 1972, Q. Appl. Math., 29, 525.

24. Ericksen, J.L., 1984, Phase Transformations and Material Instabilities in Solids, edited by M. Gurtin, (New York: Academic Press), pp. 61-77.

25. Miller, R., R. Phillips, G. Beltz and M. Ortiz, 1996, unpublished.

26. Bulatov, V. and E. Kaxiras, 1996, to be submitted to Phys. Rev. Lett.

27. Tadmor E.B., M. Ortiz and R. Phillips, 1994, Phil. Mag. A, 73, pp 1529.

28. Tadmor, E.B., R. Phillips and M. Ortiz, 1996, Langmuir, 12, pp 4529. 